\title{
In vitro evidence consistent with an interaction between wild-type and mutant SOD1 protein associated with canine degenerative myelopathy
}

\begin{abstract}
Canine degenerative myelopathy (DM) is a progressive neurological disorder that may be considered to be a large animal model for specific forms of the fatal human disease, familial amyotrophic lateral sclerosis (fALS). DM is associated with a $1118 \mathrm{G}>\mathrm{A}$ mutation of the superoxide dismutase 1 (Sod1) gene, and a significant proportion of cases are inherited in an autosomal recessive manner in contrast to the largely, but not exclusively, dominant mode of inheritance in fALS. The consensus view is that these Sod1/SOD1 mutations result in a toxic gain of function but the mechanisms remain unclear. Here we used an in vitro neuroblastoma cell line transfection system to monitor wild-type and mutant forms of SOD1 fusion proteins containing either a Cherry or an enhanced green fluorescent protein (EGFP) tag. These fusion proteins retained SOD1 enzymatic activity on a native gel assay system. We demonstrate that SOD1 aggregate density is significantly higher in DM transfectants compared to wild-type. In addition, we show by co-immunoprecipitation and confocal microscopy, evidence for a potential interaction between wild-type and mutant forms of SOD1 in cotransfected cells. While in vitro studies have shown SOD1 heterodimer formation in fALS models, this is the first report for DM SOD1. Therefore, despite for the majority of cases there is a difference in the mode of inheritance between fALS and DM, a similar interaction between wild-type and mutant SOD1 forms can occur. Clarifying the role of SOD1 in DM may also be of benefit to understanding the role of SOD1 in fALS.
\end{abstract}

Keywords: Canine; fALS; Superoxide dismutase; Aggregates; Cell toxicity 